\title{
An introduction to hearing loss and screening procedures for behavioral research
}

\author{
JAMIE REILLY \\ University of Florida, Gainesville, Florida \\ Vanessa Troiani and Murray Grossman \\ University of Pennsylvania, Philadelphia, Pennsylvania \\ AND \\ ARTHUR WingField \\ Brandeis University, Waltham, Massachusetts
}

\begin{abstract}
Hearing loss is a confounding variable that is rarely addressed in behavioral research despite its prevalence across the life span. Currently, the most common method of experimental control over hearing acuity is through self report of perceived impairment. We argue that this technique may lack sensitivity and that researchers should more commonly utilize standardized hearing screening procedures. Distinctive patterns of hearing loss are reviewed with attention to populations that commonly participate in behavioral research. We explain standard techniques for conducting pure tone hearing screening using a conventional portable audiometer and outline a procedure for how researchers can modify a conventional laptop computer for audiometric screening when a standard audiometer is unavailable. We offer a sample hearing screening program that researchers may use toward the development of their own protocol. This program is freely available for download at www .psychonomic.org/archive.
\end{abstract}

Behavioral research is typically conducted with the aim of isolating cognitive phenomena. Yet, the ability to capture central processes often depends on the assumption of intact sensation. Hearing acuity is one such factor that has gone largely uncontrolled in behavioral research. A review of the ten most recent studies that utilized auditory stimuli revealed that all controlled for the effects of hearing loss through participants' self-report of perceived impairment (Brumback, Low, Gratton, \& Fabiani, 2005; Conway \& Christiansen, 2005; Davis, Johnsrude, Hervais-Adelman, Taylor, \& Metttigan, 2005; Goldstein et al., 2005; Hughes \& Jones, 2005; Hughes, Vachon, \& Jones, 2005; Johnstone, Pleffer, Barry, Clarke, \& Smith, 2005; Moore, Bondi, Salmon, \& Murphy, 2005; Remijn \& Nakajima, 2005; Schriefers, Jeschneniak, \& Hantsch, 2005).

Report of one's metasensory experience, while informative, may lack the objectivity necessary for experimental control. This is especially so because of the tendency among individuals with mild hearing loss to be unaware of, and hence underreport, an actual hearing loss (e.g., Erdman, 1994). Populations especially vulnerable to this confound include: young children, young adults who experience transient noise exposure, older adults who experience gradual hearing loss, and patients with cognitive and/or linguistic impairment (Katz, 2002).
Numerous experimental paradigms are susceptible to the effects of hearing loss. Yet, few options exist for researchers without formal training in audiometric assessment. Our aims here are twofold; first, to increase awareness among researchers of the potentially confounding effects of hearing loss, and second, to describe a standard method for conducting a hearing screening in a laboratory setting.

\section{Measurement of Hearing}

Threshold-based measures of hearing most commonly examine the relation between two properties of sound: intensity and frequency. The perceptual manifestation of frequency is pitch, which is typically reported in Hertz (Hz) equivalent to cycles per second. The audible frequency range of human hearing has been estimated as between 20 to $20,000 \mathrm{~Hz}$, with the ear most sensitive to frequencies from 500 to $4,000 \mathrm{~Hz}$, the range that includes the general frequencies of human speech (Newby \& Popelka, 1992).

Intensity, perceived as volume, reflects the relative amplitude of a sound wave. Unlike frequency, there is an imperfect correspondence between perceived intensity and waveform amplitude across the frequency spectrum. For example, two sounds that differ by frequency (e.g., a hummingbird and a refrigerator motor) but are identical with respect to sound pressure level (i.e., waveform amplitude) 
are perceived at different volumes. The decibel $(\mathrm{dB})$ is, therefore, a relative scale that reflects either sound pressure level (SPL) or hearing level (HL). For purposes of hearing assessment, the decibel is typically reported on the HL scale. Figure 1 represents the relation between intensity and frequency along with the distribution of phonemes across the frequency range.

The signal-to-noise ratio is mediated by the integrity of the auditory pathway before auditory detail is available for cortical processing. Hearing acuity is, therefore, strongly correlated with changes in auditory physiology across the life span. Hearing impairment that results from structural dysfunction prior to sound reaching the cochlea is collectively referred to as conductive loss. Conductive loss may result from factors such as a collapsed outer ear canal, rigidity of the tympanic membrane, or middle ear infection. In contrast, hearing loss whose etiology lies within or beyond the cochlea is referred to as sensorineural in nature.

Typical age-related hearing loss is sensorineural and associated with a distinctive set of symptoms. These include difficulties perceiving speech in noisy environments and high frequency sounds in isolation (Brant \& Fozard, 1990; Corso, 1963; Gates, Cooper, Kannel, \& Miller, 1990; Kochkin, 2005; Kryter, 1983; Moscicki, Elkins, Baum, \& McNamara, 1985; Robinson, 1988; Robinson \& Sutton, 1979; Spoor, 1967). People who experience high-frequency loss may have difficulty perceiving environmental noise such as a watch ticking, a bee buzzing, or a telephone ringing. They may also have trouble making distinctions between phonemes with formants differentiated by high frequency energy (Turner \& Cummings, 1999).

Hearing deficits are more likely to impact experimental paradigms where words or sounds appear in isolation. For example, during an auditory lexical decision task participants discriminate words from nonwords; a partici- pant with high-frequency loss may perceive the word fish presented in isolation as a degraded nonword stimulus, / fi-\#/, thus suggesting a central phonological or lexical impairment. Effects are often subtle and may escape conscious awareness. Furthermore, the prevalence of hearing impairment in older adults and increasing incidence of noise-induced hearing loss among younger adults underscores the need for control over this essential variable.

It would be ideal to have full audiometric testing conducted by an audiologist to affirm the presence of normal hearing so as to exclude potential participants who may have a hearing loss. Complete audiometric testing is designed to detect not merely the presence of a hearing loss but the degree of hearing loss and its likely etiology. Such testing will typically include measurement of pure tone thresholds (the lowest intensity of sound that can be reliably detected at each tested frequency) using both air and bone conduction. Full audiometric testing will also typically include measurement of speech reception thresholds (SRTs) with and without noise, tympanometry to assess middle ear function, distortion-product otoacoustic emissions (DPOAEs) as an index of cochlear outer hair cell loss, and often auditory brainstem responses and other sophisticated measures (Katz, 2002). Clinically normal hearing for speech is often defined as a pure tone threshold average (PTA) of $25 \mathrm{~dB}$ or less for the critical frequencies for speech $(500,1000,2000$, and $4000 \mathrm{~Hz}$; Hall \& Mueller, 1997).

It is the case that many researchers will not have available to them a clinical audiometer for determination of exact hearing thresholds nor the possibility of full audiometric testing conducted by a trained audiologist. The screening test we outline below is not designed to substitute for a full audiometric workup, but rather, as a quick and accessible method of excluding potential participants whose hearing may be questionable in a more objective manner than participants' personal reports.

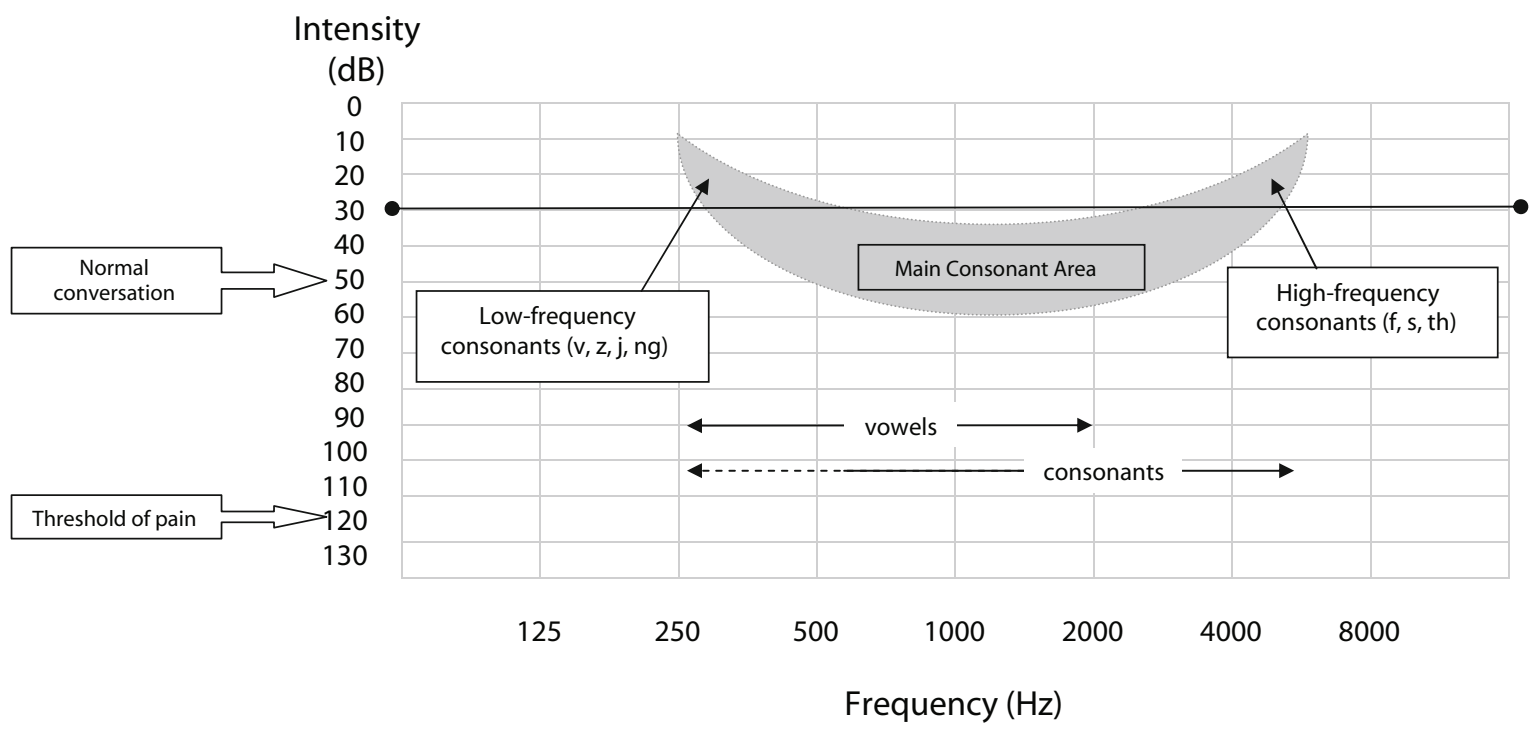

Figure 1. Relative intensity and distribution of dominant frequencies of phonemes. 


\section{Standard Pure Tone Hearing Screening}

The purpose of screening is gross identification or detection rather than fine-grained assessment. Although screening procedures are moderately sensitive to hearing loss, they have poor diagnostic specificity. That is, it is impossible to discern etiology when an individual fails to detect a specific tone, and researchers should be clearly cautioned against doing so. Whereas hearing screening procedures typically have poor specificity, they do appear to be sensitive toward identifying individuals with hearing loss (Nadol, 1993). ${ }^{1}$ In behavioral research, this degree of sensitivity may be useful toward "catching" participants whose performance may compromise the results of an experiment.

Standard pure tone hearing screening typically involves detection of tones that sweep critical points along the frequency range of speech perception (ASHA, 1996). The most common frequencies tested are those that sample in the frequency range of 500 to $4000 \mathrm{~Hz}$.

For adult participants to pass a pure tone hearing screening, they must successfully identify tones presented monaurally to either ear at $25 \mathrm{~dB} \operatorname{HL}(1000,2000$, and $4000 \mathrm{~Hz}$ ), followed by presentation of the same frequency tones to the contralateral ear. Tones should be presented at staggered intervals to prevent detection based on an underlying pattern of stimulus delivery. The participant should be seated with no direct view of the audiometer and should be instructed to signal their detection of a tone using a definitive response (e.g., raising a hand).

The order of tone presentation should be randomized within ear but not between, i.e., sweep one ear completely followed by the other. If a participant does not detect a specific tone, the same tone should be presented again after a staggered time interval. If the participant fails to identify the second presentation of a tone, they have failed the screen. This result can then be reported in a researcher's respective Method section. ${ }^{2}$

In contrast with clinical audiometric testing conducted in a commercial sound isolated audiometric testing chamber, laboratories of many behavioral researchers contain ambient noises such as computer fans. For this reason, and because various headphone manufacturers and models have differences in output intensities and frequency response, it is important that the experiments be conducted in the same room and using the same equipment as used for the initial hearing screen. This emphasizes the point that the 25-dB HL threshold is a sound level relative to the ambient listening conditions. This is a major distinction between this screening procedure for the purposes of participant selection and clinical audiometric testing where threshold levels are calibrated to a shared standard.

\section{Materials}

In the section to follow, we outline a procedure for modification of a laptop computer to achieve comparable sensitivity at near ANSI specification (ANSI S3.6-1996) for conducting audiometric screening. We developed a pure tone auditory screening protocol for use on Windowsbased operating systems running E-Prime software
(Schneider, Eschman, \& Zuccolotto, 2002). Although this screening program is designed for one specific presentation program, it is adaptable to other stimulus delivery programs such as PsyScope (Cohen, MacWhinney, Flatt, \& Provost, 1993) and Presentation (Neurobehavioral Systems, 2002) using the procedures described here. The complete screening program is available for download from www.psychonomic.org/archive.

Pure tone wavefiles were created using a 16-bit, $44100-\mathrm{Hz}$ sampling rate using the $\mathrm{NCH}$ Pure Tone generator (www.nch.com.au/tonegen). This application was used to specify frequency and duration of each wavefile. The Praat waveform editor (Boersma \& Weenink, 1996) was used to insert silences into each tone to create tone bursts. Although many audiologists use continuous tones in audiometric testing, pulsed tones as employed in this protocol have similar test-retest reliability and incidence of false positives as continuous tones. Pulsed tones have several advantages, however. These advantages include increased tone awareness and reduced vulnerability to internal or external noise (Burk \& Wiley, 2004; Martin \& Clark, 2006, p. 80).

Wavefiles were created in stereo (dual channel) format to assess left and right ear tone detection separately. Pure tone wavefiles were created at 1000,2000 , and $4000 \mathrm{~Hz}$ with $25-\mathrm{dB}$ intensity pulsed only to the left channel to assess left ear function. Similarly, three analogous wavefiles were created that pulsed sound to the right channel to assess right ear function.

We standardized wavefile volume using the Praat wavefile editor (Boersma \& Weenink, 1996) to emit a 25-dB tone ( \pm 2 -dB error) at a computer's maximum volume presented over stereo headphones. Intensity readings were obtained for these pure tones delivered by a Dell Inspiron laptop computer using stereo headphones (Sennheiser Electronic Corporation) that completely cover the ear. Intensity was measured by threading a fiberoptic probe microphone (Audioscan Verifit RM500) through the author's ear canal until the microphone rested against the tympanic membrane. This procedure allowed for precise measurement of sound intensity at the eardrum.

Using this calibration procedure, we consistently obtained intensity readings within $25 \pm 2 \mathrm{~dB}$ HL. Upon repeated measurement, variance due to frequency spectral smearing and intensity were well within ANSI accepted standards for screening audiometry (ANSI S3.6-1996).

\section{Screening Structure}

Instructions for running this screening program via E-Prime are summarized in the Appendix. Participants are first familiarized to the signaling procedure (i.e., space bar press) by identifying a tone presented binaurally at a comfortable hearing level ( $>60 \mathrm{~dB} \mathrm{HL})$. Upon successful completion of the familiarization sequence, participants complete the actual screening portion of the program.

For participants to pass this screening, they must successfully identify tones first presented monaurally to the left ear at $25 \mathrm{~dB} \mathrm{HL}(1000,2000$, and $4000 \mathrm{~Hz})$, followed by presentation of the same frequency tones to the right ear. Tones are presented at random interstimulus intervals 


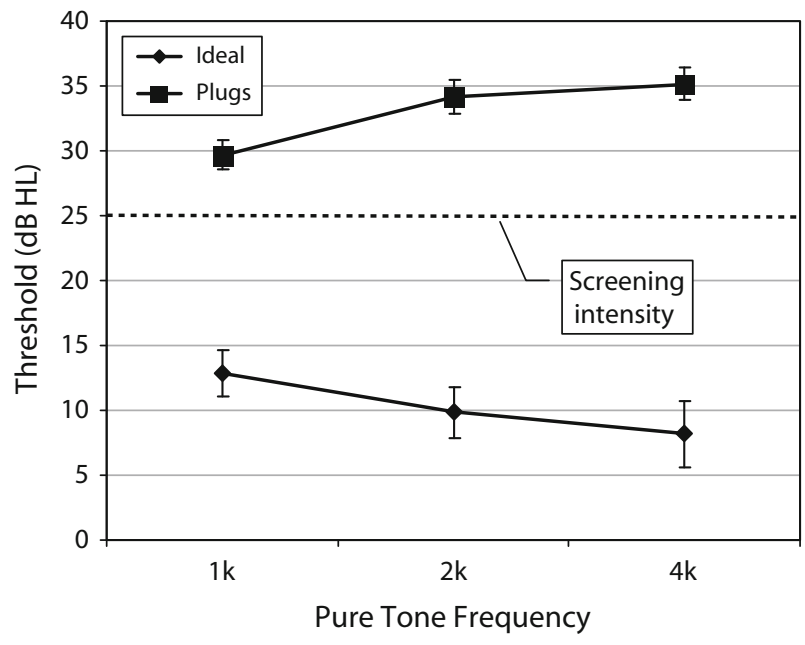

Figure 2. Unmasked air conduction thresholds at 1, 2, and $4 \mathrm{kHz}$ for ideal listening and with a simulated conductive hearing loss.

ranging from 4 to $14 \mathrm{sec}$ in $1-\mathrm{sec}$ increments. If a participant fails to detect a particular tone, the identical tone is presented again after a random interval between 4 and $14 \mathrm{sec}$. If a participant is unable to detect this repeated presentation, they have failed the screen. If a participant identifies all pure tones presented at 25-dB HL binaurally, they have passed this screen.

Throughout the duration of the screening program participants view an unchanging monitor display on which appears a centered graphic of set of headphones with the following instructions centered below in 22-point font: "Listen carefully" and "Press the space bar when you hear a tone." E-Prime was programmed to count a keypress within 3,000 msec of a tone onset as correct. If participants do not press the space bar within 3,000 msec, E-prime registers no response as a miss. False positives (i.e., space bar press when no signal is present) are logged in the data file but not treated as misses. Although this program is designed for E-Prime, the protocol is adaptable to other presentation programs using the methods described here (see appendices). The scripts associated with this screening protocol, written in visual basic script, are also downloadable from www.psychonomic.org/archive. For researchers without access to a stimulus delivery program such as E-Prime or PsyScope, tones may also be presented manually using a standard media player.

The implementation of this particular program will require calibration. That is, the frequency and intensities we obtained with our particular headphone set and computer are unique. Different stereo headphones and soundcards are likely to produce different results. Therefore, it is necessary to calibrate one's particular equipment to produce tones of the specified frequency and intensity. This calibration may be achieved through the procedure described previously (i.e., probe microphone in ear). Although probe microphones are not standard equipment in behavioral laboratories, access to this equipment is not difficult to achieve. Probes are standard equipment in most audiology and otolaryngology clinics, hearing aid dispensers, and in university speech and hearing departments.

\section{Pilot Testing}

We screened fifteen young adults from the University of Pennsylvania community (mean age $=28.93$ years, $S D=$ 4.74 , range $=22-36$ ) using both the E-Prime program and a conventional portable audiometer (Micro Audiometrics DSP) in both a quiet condition (ideal) and also with a simulated binaural conductive hearing loss. This loss was simulated by inserting foam earplugs (rated at 29-dB sound attenuation) into both ears and then occluding the plugged ears with headphones. Order of administration of screening tool (audiometer or PC) was counterbalanced. Hearing threshold was established for the lowest audible tone detectable binaurally at 1,2 , and $4 \mathrm{kHz}$ using the portable audiometer under the normal condition (no earplugs) and with a simulated conductive hearing loss. Thresholds were obtained by presenting tones at a comfortable volume ( $50 \mathrm{~dB} \mathrm{HL})$ and then descending in $10-\mathrm{dB}$ increments until the participant failed to detect a tone. Intensity was then increased in 5-dB increments until the participant successfully detected the tone upon two independent ascending presentations. Figure 2 represents group hearing thresholds under a normal screening condition and with a simulated conductive hearing loss.

To examine the correlation of this PC-based screening program with the conventional audiometer, the following factors were crossed: (1) ear (left or right); (2) tone frequency $(1,2$, or $4 \mathrm{kHz}$ ); (3) simulated loss (yes/no); (4) screening tool (audiometer or E-Prime program). This design produced 24 observations per participant, for a total of 360 observations, presented in Table 1.

\section{Results}

The portable audiometer and PC program demonstrated similar screening sensitivity under both the normal testing condition [percentage agreement between screening tools $=96.67 \%$; Cochran's $\mathrm{Q}=.33, d f=1, p=.56]$ and with a simulated conductive hearing loss [percentage agreement $=83.33 \%$; Cochran's $\mathrm{Q}=1.67, d f=2, p=$ .20]. Of note, two of fifteen participants who initially reported normal hearing acuity failed to detect tones at one or more frequencies with both audiometry and the PCbased program.

\section{Discussion}

Hearing screening is advantageous in that it provides a rapid means of control over a variable known to affect performance on many auditory paradigms (e.g., Wingfield, Tun, \& McCoy, 2005). Whereas formal diagnostic assessment of hearing is not within the scope of experimental psychology, control remains a cornerstone of the scientific method. Therefore, we argue that researchers who use auditory stimuli should better familiarize themselves with the potential impact of hearing loss and procedures for screening that move beyond self-report. There are many commercially available portable audiometers that may easily be integrated 
Table 1

Results of Pilot Data

\begin{tabular}{|c|c|c|c|c|c|c|c|c|c|c|c|c|c|}
\hline \multirow[b]{3}{*}{ Subject } & \multirow{3}{*}{$\begin{array}{l}\text { Listening } \\
\text { Condition }\end{array}$} & \multicolumn{6}{|c|}{ Left Ear Frequencies (kHz) } & \multicolumn{6}{|c|}{ Right Ear Frequencies (kHz) } \\
\hline & & \multicolumn{2}{|c|}{1} & \multicolumn{2}{|c|}{2} & \multicolumn{2}{|c|}{4} & \multicolumn{2}{|c|}{1} & \multicolumn{2}{|c|}{2} & \multicolumn{2}{|c|}{4} \\
\hline & & Aud & $\mathrm{PC}$ & Aud & $\mathrm{PC}$ & Aud & $\mathrm{PC}$ & Aud & $\mathrm{PC}$ & Aud & $\mathrm{PC}$ & Aud & $\mathrm{PC}$ \\
\hline \multirow[t]{2}{*}{1} & Normal & 1 & 1 & 1 & 1 & 1 & 1 & 1 & 1 & 1 & 1 & 1 & 1 \\
\hline & Simulated loss & 1 & 1 & 1 & 1 & 1 & 1 & 0 & 1 & 0 & 1 & 0 & 0 \\
\hline \multirow[t]{2}{*}{2} & Normal & 1 & 1 & 1 & 1 & 1 & 1 & 1 & 1 & 1 & 1 & 1 & 1 \\
\hline & Simulated loss & 1 & 1 & 1 & 1 & 0 & 1 & 1 & 1 & 1 & 1 & 0 & 0 \\
\hline \multirow[t]{2}{*}{3} & Normal & 1 & 1 & 1 & 1 & 1 & 1 & 1 & 1 & 1 & 1 & 1 & 1 \\
\hline & Simulated loss & 1 & 1 & 1 & 1 & 1 & 1 & 1 & 1 & 1 & 1 & 1 & 1 \\
\hline \multirow[t]{2}{*}{4} & Normal & 1 & 1 & 1 & 1 & 1 & 1 & 1 & 1 & 1 & 1 & 1 & 1 \\
\hline & Simulated loss & 1 & 1 & 0 & 0 & 1 & 1 & 1 & 1 & 1 & 1 & 1 & 1 \\
\hline \multirow[t]{2}{*}{5} & Normal & 1 & 1 & 1 & 1 & 1 & 1 & 1 & 1 & 1 & 1 & 1 & 1 \\
\hline & Simulated loss & 1 & 1 & 1 & 1 & 1 & 1 & 1 & 1 & 1 & 1 & 1 & 1 \\
\hline \multirow[t]{2}{*}{6} & Normal & 1 & 1 & 1 & 1 & 1 & 1 & 1 & 1 & 1 & 1 & 1 & 1 \\
\hline & Simulated loss & 1 & 1 & 0 & 1 & 1 & 1 & 1 & 1 & 1 & 1 & 0 & 0 \\
\hline \multirow[t]{2}{*}{7} & Normal & 1 & 1 & 1 & 1 & 1 & 1 & 1 & 1 & 1 & 1 & 1 & 1 \\
\hline & Simulated loss & 1 & 1 & 1 & 1 & 0 & 0 & 1 & 0 & 1 & 0 & 0 & 1 \\
\hline \multirow[t]{2}{*}{8} & Normal & 1 & 1 & 1 & 1 & 1 & 1 & 1 & 1 & 1 & 1 & 1 & 1 \\
\hline & Simulated loss & 0 & 1 & 0 & 0 & 0 & 1 & 0 & 0 & 0 & 0 & 0 & 0 \\
\hline \multirow[t]{2}{*}{9} & Normal & 1 & 1 & 1 & 1 & 1 & 1 & 1 & 1 & 1 & 1 & 1 & 1 \\
\hline & Simulated loss & 1 & 1 & 1 & 1 & 1 & 1 & 1 & 1 & 1 & 1 & 1 & 1 \\
\hline \multirow[t]{2}{*}{10} & Normal & 1 & 1 & 1 & 1 & 1 & 1 & 1 & 1 & 1 & 1 & 1 & 1 \\
\hline & Simulated loss & 0 & 1 & 0 & 1 & 0 & 1 & 0 & 0 & 0 & 0 & 0 & 0 \\
\hline \multirow[t]{2}{*}{11} & Normal & 1 & 1 & 1 & 1 & 1 & 1 & 1 & 1 & 1 & 1 & 1 & 0 \\
\hline & Simulated loss & 1 & 1 & 0 & 1 & 0 & 1 & 1 & 1 & 1 & 1 & 1 & 1 \\
\hline \multirow[t]{2}{*}{12} & Normal & 1 & 1 & 1 & 1 & 1 & 1 & 1 & 1 & 1 & 1 & 1 & 1 \\
\hline & Simulated loss & 0 & 1 & 0 & 0 & 1 & 1 & 0 & 1 & 0 & 1 & 0 & 0 \\
\hline \multirow[t]{2}{*}{13} & Normal & 1 & 1 & 1 & 1 & 1 & 1 & 1 & 1 & 1 & 1 & 1 & 1 \\
\hline & Simulated loss & 0 & 1 & 0 & 0 & 0 & 0 & 0 & 0 & 0 & 0 & 0 & 0 \\
\hline \multirow[t]{2}{*}{14} & Normal & 0 & 1 & 1 & 1 & 1 & 1 & 1 & 1 & 1 & 1 & 1 & 1 \\
\hline & Simulated loss & 1 & 1 & 0 & 1 & 0 & 0 & 1 & 1 & 0 & 1 & 0 & 1 \\
\hline \multirow[t]{2}{*}{15} & Normal & 0 & 0 & 0 & 0 & 0 & 1 & 0 & 0 & 0 & 0 & 1 & 0 \\
\hline & Simulated loss & 0 & 1 & 0 & 0 & 0 & 0 & 0 & 0 & 0 & 0 & 0 & 0 \\
\hline
\end{tabular}

Note-1, pass; 0 , fail.

into a laboratory setting. Furthermore, we have described a unique method for modification of a PC to obtain comparable sensitivity to screening audiometry. We encourage researchers to contact the authors for technical assistance.

\section{AUTHOR NOTE}

This work was supported in part by NIH/NRSA Postdoctoral Training Grant AG00255 awarded to the first author from the National Institute of Aging. We also acknowledge support from Grant R01 AG19714 from the National Institute of Aging. We are grateful for the assistance of Kevin Frank, Joseph Donaher, and the audiology departments of the Children's Hospital of Philadelphia and Mercy Fitzgerald Hospital. Correspondence concerning this article should be addressed to J. Reilly, Departments of Communicative Disorders and Neurology, University of Florida, P.O. Box 100174, Gainesville, FL 32610 (e-mail: jjreilly@ phhp.ufl.edu).

\section{REFERENCES}

American National Standards Institute (1996). Specification for Audiometers. ANSI, S3.6-1996, New York: Author.

American Speech-Language-Hearing Association (1996). Guidelines for audiologic screening. Rockville, MD: Author.

Boersma, P., \& Weenink, D. (1996). Praat, a system for doing phonetics by computer (Version 3.4, Report 132). University of Amsterdam, Institute of Phonetic Sciences.

BRANT, L. J., \& FozARD, J. L. (1990). Age changes in pure-tone hearing thresholds in a longitudinal study of normal human aging. Journal of the Acoustical Society of America, 88, 813-820.

Brumback, C. R., Low, K. A., Gratton, G., \& Fabiani, M. (2005).
Putting things into perspective: Individual differences in workingmemory span and the integration of information. Experimental Psychology, 52, 21-30.

Burk, M. H., \& WiLEY, T. L. (2004). Continuous versus pulsed tones in audiometry. American Journal of Audiology, 13, 54-61.

Cohen, J. D., MacWhinney, B., Flatt, M., \& Provost, J. (1993). PsyScope: An interactive graphic system for designing and controlling experiments in the psychology laboratory using Macintosh computers. Behavior Research: Methods, Instruments, \& Computers, 25, 257-271.

Conway, C. M., \& Christiansen, M. H. (2005). Modality-constrained statistical learning of tactile, visual, and auditory sequences. Journal of Experimental Psychology: Learning, Memory, \& Cognition, 31, 24-39.

Corso, J. F. (1963). Age and sex differences in pure-tone thresholds. Arch Otolaryngologica, 77, 385-405.

Davis, M. H., Johnsrude, I. S., Hervais-Adelman, A., Taylor, K., \& McGetTigan, C. (2005). Lexical information drives perceptual learning of distorted speech: Evidence from the comprehension of noise-vocoded sentences. Journal of Experimental Psychology: General, 134, 222-241.

ERDMAN, S. A. (1994). Self-assessment: From research focus to research tool. Journal of the Academy of Rehabilitative Audiology Monographs, 27, 67-90.

Gates, G. A., Cooper, J. C., Kannel, W. B., \& Miller, N. J. (1990). Hearing in the elderly: The Framingham cohort: Part 1. Basic audiometric test results. Ear Hear, 11, 247-256.

Goldstein, J. M., Jerram, M., Poldrack, R., Anagnoson, R., Breiter, H. C., MAKRIS, N., ET AL. (2005). Sex differences in prefrontal cortical brain activity during fMRI of auditory verbal working memory. Neuropsychology, 19, 509-519.

Hall, J., \& Mueller, G. (1997). Audiologist desk reference. San Diego: Singular. 
Hughes, R. W., Jones, D. M. (2005). The impact of order incongruence between a task-irrelevant auditory sequence and a task-relevant visual sequence. Journal of Experimental Psychology: Human Perception \& Performance, 31, 316-327.

Hughes, R. W., Vachon, F., \& Jones, D. M. (2005). Auditory attentional capture during serial recall: Violations at encoding of an algorithmbased neural model? Journal of Experimental Psychology: Learning, Memory, \& Cognition, 31, 736-749.

Johnstone, S. J., Pleffer, C. B., Barry, R. J., Clarke, A. R., \& Smith, J. L. (2005). Development of inhibitory processing during the go/no-go task: A behavioral and event-related potential study of children and adults. Journal of Psychophysiology, 19, 11-23.

Katz, J. (2002). Handbook of clinical audiology (5th ed.). Philadelphia: Lippincott Williams \& Wilkins.

KochKIN, S. (2005). MarkeTrak VII: Hearing loss population tops 31 million people. Hearing Review, 12, 16-29.

Kryter, K. D. (1983). Presbycusis, sociocusis and nosocusis. Journal of the Acoustical Society of America, 73, 254-258.

Martin, F. N., \& Clark, J. G. (2006). Introduction to audiology (9th ed.). Boston: Pearson.

Moore, A. B., Bondi, M. W., Salmon, D. P., \& Murphy, C. (2005). Eyeblink classical conditioning to auditory and olfactory stimuli: Performance among older adults with and without the apolipoprotein $\mathrm{E}$ $\varepsilon 4$ allele. Neuropsychology, 19, 437-445.

Moscicki, E. K., Elkins, E. F., Baum, H. M., \& McNamara, P. M. (1985). Hearing loss in the elderly: An epidemiological study of the Framingham heart study cohort. Ear Hear, 6, 184-190.

NAdOL, J. (1993). Hearing loss. New England Journal of Medicine, 329, 1092-1102.

NCH Pure Tone Generator. Accessed December 1, 2005. Available at www.nch.com.au/tonegen.

Newby, H. A., \& Popelka, G. R. (1992). Audiology (6th ed.). Englewood Cliffs, NJ: Prentice Hall.

Remisn, G. B., \& NaKajima, Y. (2005). The perceptual integration of auditory stimulus edges: An illusory short tone in stimulus patterns of consisting of two partly overlapping glides. Journal of Experimental Psychology: Human Perception \& Performance, 31, 183-192.

Robinson, D. W. (1988). Threshold of hearing as a function of age and sex for the typical unscreened population. British Journal of Audiology, 22, 5-20.
Robinson, D. W., \& Sutton, G. J. (1979). Age effect in hearing: A comparative analysis of published threshold data. Audiology, 18, 320-304.

Schneider, W., Eschmann, A., \& Zuccolotto, A. (2002). E-Prime Reference Guide. Pittsburgh: Psychology Software Tools.

Schriefers, H., Jescheniak, J. D., \& Hantsch, A. (2005). Selection of gender-marked morphemes in speech production. Journal of Experimental Psychology: Learning, Memory, \& Cognition, 31, 159-168.

SPOOR, A. (1967). Presbycusis values in relation to noise induced hearing loss. International Journal of Audiology, 6, 48-57.

Turner, C. W., \& Cummings, K. J. (1999) Speech audibility for listeners with high-frequency hearing loss. American Journal of Audiology, 8, 47-56.

Wingfield, A., Tun, P. A., \& McCoy, S. L. (2005). Hearing loss in older adulthood: What it is and how it interacts with cognitive performance. Current Directions in Psychological Science, 14, 144-148.

\section{NOTES}

1. A pure tone resonates at only one frequency with no harmonic content. Acoustic properties of speech and other environmental noises are not characterized by the simple sine wave function of a pure tone.

2. A sample statement reporting a passed hearing screening would appear as follows: Participants passed a pure tone hearing screening administered binaurally at $25 \mathrm{~dB}$ (HL) at 1000, 2000, and $4000 \mathrm{~Hz}$.

\section{ARCHIVED MATERIALS}

The following materials associated with this article may be accessed through the Psychonomic Society's Norms, Stimuli, and Data archive, www.psychonomic.org/archive/.

To access these files, search the archive for this article using the journal name (Behavior Research Methods), the first author's name (Reilly), and the publication year (2007).

FILE: Reilly-BRM-2007.zip

DESCRIPTION: The compressed archive file contains eight files:

6 wavefiles.

1 E-Prime program.

1 E-Prime Visual Basic Script file.

AUTHOR's E-MAIL ADDRESs: jjreilly@phhp.ufl.edu

\section{APPENDIX \\ Instructions for Screen Administration}

Download the hearing screening program, wavefiles, and scripts from the following Web site: www .psychonomic.org/archive

Make certain that testing is completed in a very silent environment.

Turn the system volume to maximum.

If running this program using a system other than E-Prime, make certain that the volume control for of the secondary program (e.g., Media Player) is also set to its maximum.

Consider temporarily disabling Windows Critical Stop and other system sounds that will be presented at an uncomfortable volume.

Use quality stereo headphones that cover the ear. Do not use insert headphones or present tones via an external speaker.

Remain consistent in the use of headphones and testing setting.

(Manuscript received April 30, 2006;

revision accepted for publication September 1, 2006.) 